\title{
Generation of Monoclonal Antibodies Specific for Human Hemoglobin
}

\author{
Hajime Yoshida* and Akira KuSANO \\ Tokyo Research Laboratories, Kyowa Hakko Kogyo Co., Ltd., \\ 3-6-6 Asahi-machi, Machida-shi, Tokyo 194, Japan
}

Received June 6, 1988

\begin{abstract}
One monoclonal antibody (MoAb) highly specific for human hemoglobin, KM-371, was generated by a unique immunization procedure using mice rendered tolerant to bovine hemoglobin.

KM-371 strongly reacted with human hemoglobin, but did not with bovine, horse, goat, sheep, rabbit, porcine, chicken, and fish hemoglobins. Human hemoglobin was able to be quantitatively measured in the range $0.01 \sim 200 \mu \mathrm{g} / \mathrm{ml}$ by a sandwich ELISA using KM-371 and biotinylated KM371. By immunostaining using KM-371, $20 \mathrm{ng}$ of human hemoglobin could be detected on a nitrocellulose membrane.

These results suggest that $\mathrm{KM}-371$ would be a useful MoAb in the test of occult bloods in faeces for diagnosing colorectal carcinoma without a meat diet.
\end{abstract}

The test of faeces for occult bloods is the most effective and confidential diagnosis for colorectal carcinoma in its early stage. Commercial diagnostic kits for occult bloods are based on a chemical reaction that is catalysed by hemoglobin contained in the blood. Recently, the use of polyclonal and monoclonal antibody against hemoglobin to detect occult bloods in faeces has been studied. Dietary attention before the check is usually neccessary, because these methods lack specificity to human hemoglobin.

We previously reported the generation of anti-human tumor MoAbs using mice rendered tolerant to normal human tissues. $\left.{ }^{\sim} \sim 3\right)$

In the present paper, we describe the generation of MoAbs specific for human hemoglobin using mice rendered tolerant to animal's hemoglobin, and theier characterization.

\section{MATERIALS AND METHODS}

Hemoglobins. Porcine, chicken, and fish hemoglobin were prepared in our laboratory from meat available on the market. ${ }^{4}$ Human hemoglobin was purchased from Nutritional Biochemicals (U.S.A.), and bovine, horse, goat, sheep, and rabbit hemoglobin were purchased from Sigma (U.S.A.).

Acquisition of tolerance and immunization. Newborn BALB/c mice were rendered tolerant by i.p. and s.c. injections of $1 \mathrm{mg}$ of protein of bovine hemoglobin within $24 \mathrm{~h}$ after birth. The tolerant mice were then immunized by i.p. injections of $0.1 \mathrm{mg}$ of human hemoglobin mixed with aluminum gel and Bordetella pertusis as an adjuvant at $6 \sim 8$ weeks old. Two booster i.p. injections of the same amount of human hemoglobin were given at intervals of 1 week.

Production of hybridomas. Mice which showed high reactivity to human hemoglobin and low reactivity to bovine hemoglobin were sacrificed 4 days after the last booster injection. Splenocytes were fused with a mouse myeloma cell line, P3-X63. Ag8-U1(P3U1), using polyethylene glycol 1000 by a modified method of Köhler and Milstein. ${ }^{51}$ Fused cells were distributed into 96-well culture plates in hypoxanthine: aminopterine: thymidine medium, and antibody production was examined by ELISA after a 10- to 12-day incubation. The cells were subsequently cloned twice by limiting dilution in 96-well

* To whom requests for reprints should be addressed.

Abbrevations: ELISA, enzyme-linked immunosorbent assay; SDS, sodium dodecyl sulfate; PAGE, polyacrylamide gel electrophoresis; FCS, fetal calf serum; PBS, phosphate-buffered saline; BSA-PBS, PBS which contains $1 \%$ (w/v) bovine serum albumin; Tween-PBS, PBS which contains $0.05 \%(\mathrm{v} / \mathrm{v})$ Tween $20 ; \mathrm{MoAb}$, monocional antibody. 
culture plates.

Binding ELISA. For binding ELISA, the wells of 96well EIA plates were coated with various kinds of hemoglobins at $10 \mu \mathrm{g} / \mathrm{ml}$ in PBS overnight at $4^{\circ} \mathrm{C}$. The antigencoated plates were flooded with $1 \%$ bovine serum albumin in PBS to block the remaining protein-binding sites. Fifty $\mu \mathrm{l}$ of a sample were added to each well, and the plates incubated at room temperature for $2 \mathrm{hr}$ or overnight at $4{ }^{\circ} \mathrm{C}$. After washing 3 times with $0.05 \%$ Tween 20 in PBS, $50 \mu \mathrm{l}$ of peroxidase-labeled rabbit anti-mouse immunoglobulin (Dakopatts, Denmark) was allowed to react at room temperature for $2 \mathrm{hr}$. After washing 6 times with $0.05 \%$ Tween 20 in PBS, $50 \mu \mathrm{l}$ of $1 \mathrm{~mm}$ 2,2-azinobis(3-ethyl benzothiazoline-6-sulfonic acid)diammonium solution were added to each well, and the plates incubated at room temperature for $15 \mathrm{~min}$. The reaction was stopped with $5 \%$ sodium dodecyl sulfate solution, and the absorbance at $414 \mathrm{~nm}$ was measured by a microtiter plate analyser (NJ2000; Japan Intermed, Japan).

SDS-PAGE and western blotting. SDS-PAGE was done in $10 \%(\mathrm{w} / \mathrm{v})$ polyacrylamide gel by the method of Laemmli. ${ }^{6}$ Two micrograms of various hemoglobins were developed by SDS-PAGE. They were then transferred to nitrocellulose membranes in a transfer buffer $(0.025 \mathrm{M}$ Tris, $0.2 \mathrm{M}$ glycine, $20 \%$ methyl alcohol, $\mathrm{pH} 8.3$ ) overnight at $4^{\circ} \mathrm{C}$ by the method of Towbin et al. ${ }^{7)}$ The dried nitrocellulose membrane was soaked in a $3 \%$ gelatin solution to block the remaining protein-binding sites. Then it was dipped in culture supernatants of the hybridomas to human hemoglobin and incubated at room temperature for $2 \mathrm{hr}$. After being washed 3 times with Tween-PBS, peroxidase-labeled anti-mouse immunoglobulin was allowed to react at room temperature for $2 \mathrm{hr}$. After washing 3 times, the membrane was developed with $0.06 \%$ HRP Color Development Reagent (BIO-RAD, U.S.A.) in $0.02 \mathrm{~m}$ Tris $0.5 \mathrm{M} \mathrm{NaCl}$, $\mathrm{pH}$ 7.5. The reaction was stopped by soaking the membrane in cold water. Another membrane was stained with amido black after transfer.

Hemagglutination assay. Serially diluted MoAbs were tested for hemagglutinating activity by mixing $50 \mu \mathrm{l}$ of $\mathrm{MoAb}$ solution with $50 \mu \mathrm{l}$ of a $1 \%(\mathrm{v} / \mathrm{v})$ suspension of human erythrocytes in PBS in each well of a 96-well Uplate. After incubating at room temperature for $1 \mathrm{hr}$ the wells were examined microscopically for direct hemagglutination.

Quantification of human hemoglobin by sandwich ELISA. Fifty $\mu \mathrm{l}$ of anti-human MoAb $(10 \mu \mathrm{g} / \mathrm{ml})$ in PBS was added to each well of a 96-well EIA plate, and incubated overnight at $4^{\circ} \mathrm{C}$, and the wells were flooded with $1 \%$ BSA-PBS for $1 \mathrm{hr}$ at room temperature. Then, $50 \mu \mathrm{l}$ of human hemoglobin solution at various concentrations were added to the wells for further incubation overnight at $4^{\circ} \mathrm{C}$. The solution was aspirated, and the wells were washed with $0.05 \%$ Tween 20 in PBS. Fifty $\mu 1$ of biotinylated anti-human hemoglobin MoAb $(10 \mu / \mathrm{ml})$ in $1 \%$ BSA-PBS was added, and the wells incubated for $2 \mathrm{hr}$ at room temperature. After the wells had been thoroughtly washed again, $50 \mu \mathrm{l}$ of avidin-biotin-peroxidase complex ( $A B C$ kit) was added, and the plate incubated for $1 \mathrm{hr}$ at room temperature. After washing with $0.05 \%$ Tween 20 in PBS, $100 \mu$ l of ABTS solution was applied for $10 \mathrm{~min}$ at room temperature, and the absorbance at $414 \mathrm{~nm}$ was measured.

\section{RESULTS}

\section{Generation of MoAbs}

For generating MoAbs specific to human hemglobin, five mice were rendered tolerant with bovine hemoglobin and, 6 to 8 weeks later they were immunized with human hemoglobin. The reactivity of sera obtained from the mice 3 days after the last booster was determined to both the tolerogen (bovine hemoglobin) and immunogen (human hemoglobin) by ELISA (Fig. 1). Spleen cells were obtained from No. 5 mouse and fused with myeloma cells (P3U1). The fused cells producing MoAbs which exhibited reactivity to human

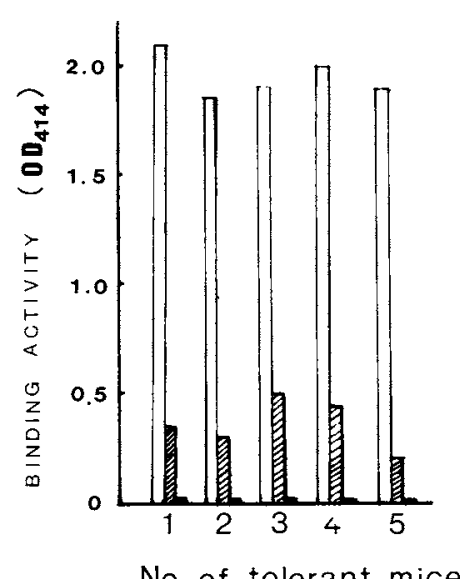

FIG. 1. Reactivity to the Tolerogen (bovine hemoglobin) and Immunogen (human hemoglobin) of Sera from Tolerant Mice by Binding ELISA.

Neonatal tolerance was induced by i.p. and s.c. injections of $1 \mathrm{mg}$ of bovine hemoglobin. Eight-week-old tolerant mice were immunized with $100 \mu \mathrm{g}$ of human hemoglobin. Sera obtained 3 days after the las booster were assayed by binding ELISA.

Key: open bar, human hemoglobin; hatched bar, bovine hemoglobin; closed bar, bovine serum albumin. 
hemoglobin and no reactivity to bovine hemoglobin were selected by ELISA. After fusion and selection, 4 clones were finally isolated. One of these MoAbs, designated KM-371, was selected for its specificity for human hemoglobin. KM-371 reacted strongly with human hemoglobin, but did not react with bovine, horse, goat, sheep, rabbit, porcine, chicken and fish hemoglobin by binding ELISA (Fig. 2).

\section{SDS-PAGE and immunoblotting}

The specificity of KM-371 was confirmed by SDS-PAGE and immunoblotting (western blotting). As shown in Fig. 3(a), all proteins were transferred to the nitrocellulose membrane under these conditions. Fig. 3(b) shows that only a single band corresponding to human hemoglobin was observed by immunostaining using KM-371.

\section{Hemagglutination assay}

KM-32, anti-human lung squamous car-

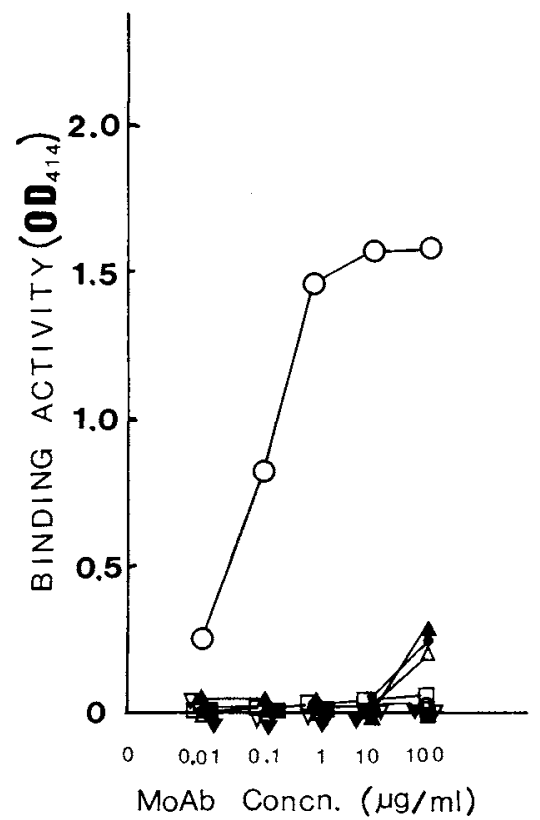

FIG. 2. Specificity of the Monoclonal Antibody, KM371 , in Binding ELISA.

Binding ELISA for KM-371 was performed against human $(\bigcirc)$, bovine $(0)$, horse $(\nabla)$, goat $(\nabla)$, sheep $(\square)$ rabbit (O), porcine $(\boldsymbol{\square})$, chicken $(\triangle)$, and fish (A) hemoglobins. cinoma MoAb, agglutinated human erythrocytes, ${ }^{11}$ whereas KM-371 did not agglutinate freshly prepared human erythrocytes (Table I).

Detection of human hemoglobin on nitrocellulose membrane

As shown in Fig. 4, more than $20 \mathrm{ng}$ of human hemoglobin on a nitrocellulose membrane was detected by immunostaining using KM371.

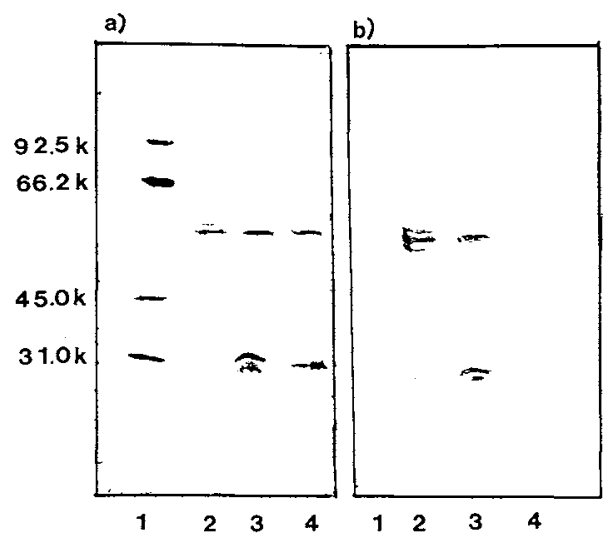

FIG. 3. Specificity of KM-371 in Western Blotting. 1, MW marker; 2, human hemoglobin (purchased from Nutritional Biochemicals); 3, human hemoglobin (freshly prepared from Human erythrocytes); 4, bovine hemoglobin (freshly prepared from bovine erythrocytes) were developed by SDS-PAGE and transferred to nitrocellulose membrane was stained with amido black (a) and another one was stained with KM-371 and the peroxidase-labeled second antibody (b).

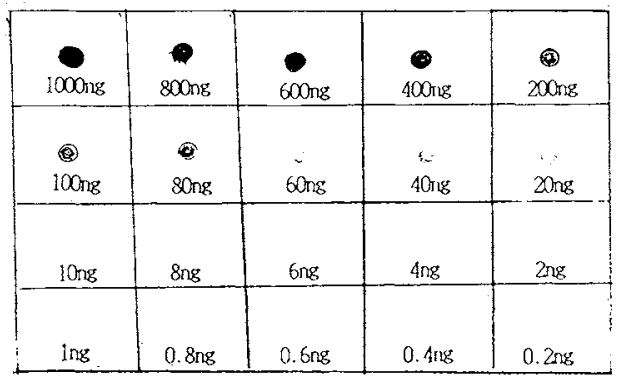

Fig. 4. Detection of Human Hemoglobin on a Nitrocellulose Membrane by KM-371.

Various concentrations of human hemoglobin were dotted on a nitrocellulose membrane. After drying, the membrane was stained with KM-371 and the peroxidase-labeled second antibody 


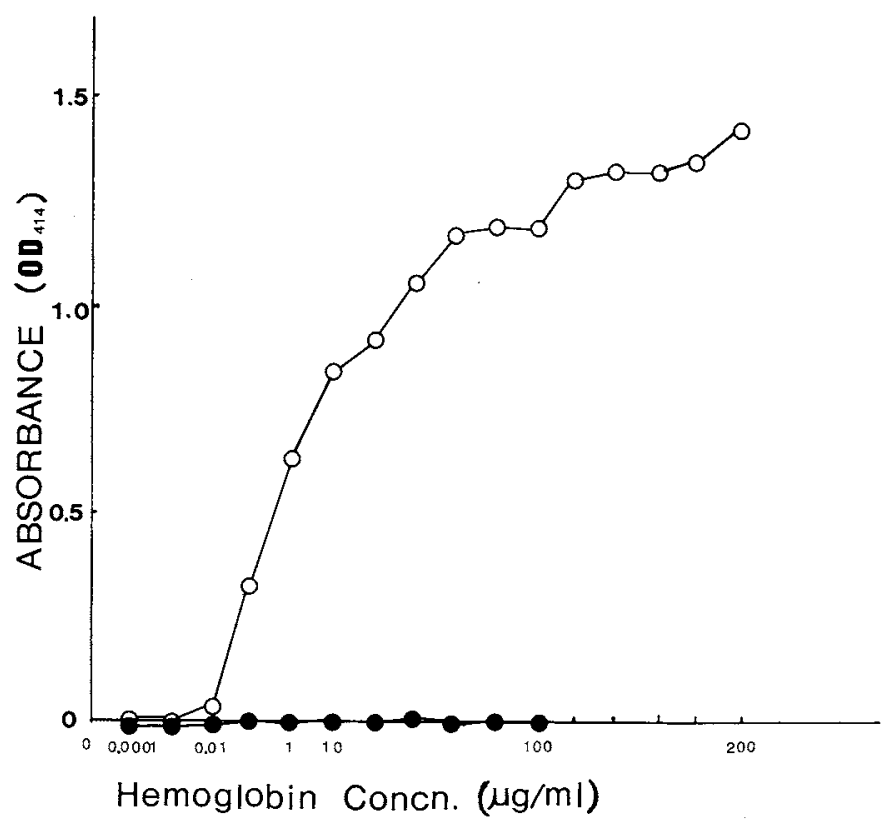

Fig. 5. Titration Curve of Human Hemoglobin by Sandwich ELISA using KM-371.

Various concentrations of human hemoglobin $(O)$ and bovine hemoglobin $(O)$ were measured by sandwich ELISA using KM-371 and biotinylated KM-371.

Table I. Hemagglutination Activity of KM-371 TOWARD HUMAN ERYTHROCTES

Experiments were carried out by the methods described in Materials and METHODS.

\begin{tabular}{ccc}
\multirow{2}{*}{$\begin{array}{c}\text { Concn. of } \mathrm{MoAb} \\
(\mu \mathrm{g} / \mathrm{ml})\end{array}$} & \multicolumn{2}{c}{ Hemagglutination activity with MoAb } \\
\cline { 2 - 3 } & $\mathrm{KM}^{2} 32^{a}$ & KM-371 \\
\hline 100 & + & - \\
10 & \pm & - \\
1 & - & - \\
0.1 & - & -
\end{tabular}

a Positive control: an anti-squamous lung carcinoma $\mathrm{MoAb}$.

\section{Quantification of human hemoglobin by sand-} wich ELISA

Quantification of human hemoglobin by sandwich ELISA, using KM371 $(101 \mu \mathrm{g} / \mathrm{ml})$ as the first antibody and biotinylated KM-371 $(1 \mu \mathrm{g} / \mathrm{ml})$ as the second antibody is shown in Fig. 5. The values of $\mathrm{OD}_{414}$ correspond well to the concentrations of human hemoglobin between $0.01 \mu \mathrm{g} / \mathrm{ml}$ and $200 \mu \mathrm{g} / \mathrm{ml}$. Animal hemoglobins (only data of bovine hemoglobin is shown in Fig. 5) were not determined in this sandwich ELISA.

\section{DISCUSSION}

The detection of occult bloods in faeces is the most sensitive and effective diagnosis of colorectal carcinoma, especially in the early stage. Recently, occult bloods in faces have been immunologically detected as hemoglobin by using anti-hemoglobin antibodies. Generally, a meat diet before the check is necessary, because these tests are not able to distinguish human hemoglobin from animal hemoglobin originating from the meat. MoAb specific for human hemoglobin will be useful for this diagnosis.

Anti-human hemoglobin MoAbs have been established and characterized by several workers, ${ }^{8,9)}$ but they were not tested for their reactivity against various animal hemoglobinorigine meats.

The homology of the amino acid sequence between human hemoglobin and that of other animals is more than $80 \%$. Therefore, it is 
difficult to established MoAbs specific for human hemoglobin by the usual hybridoma technology using pure human hemoglobin as an immunogen. We could efficiently establish a MoAb specific for human hemoglobin from mice rendered tolerant to bovine hemoglobin. KM-371, anti-human'hemoglobin MoAb established by us, reacted with only human hemoglobin, and not with their animal's hemoglobins tested. Quantification and detection of human hemoglobin by sandwich ELISA and immunostaining using KM-371 and/or biotinylated KM-371 are expected to help with the diagnosis of colorectal carcinoma without dietary attention.

\section{REFERENCES}

1) N. Hanai, K. Shitara and H. Yoshida, Cancer Res., 46, 4438 (1986).

2) N. Hanai, K. Shitara and H. Yoshida, Cancer Res., 46, 5206 (1986).

3) K. Shitara, N. Hanai and H. Yoshida, Cancer Res., 47, 1267 (1987).

4) N. Aoki and A. Shibata, "Methods in Haematology," 1980, p. 203 (in Japanese).

5) G. Köhler and C. Milstein, Nature, 256, 495 (1975).

6) U. K. Laemmli, Nature, 227, 680 (1970).

7) H. Towbin, T. Stachelin and J. Gorden, Proc. Natl. Acad. Sci., U.S.A., 76, 4350 (1979).

8) G. Stamatoyannopoulos, M. Farquhar, D. Lindsley, M. Brice, T. H. Papayannopoulou, P. E. Nute, G. R. Serjeant and H. Lehmann, The Lancet, Oct. 31, 952 (1981).

9) C. J. Krco, T. J. Gorzynski and T. Beito, J. Immunogenetics, 12, 197 (1985). 